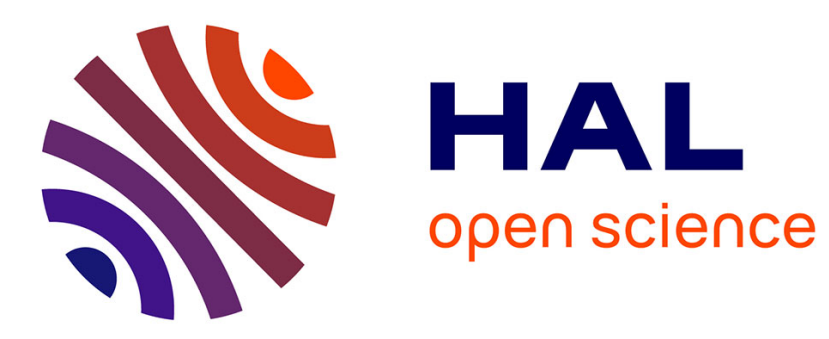

\title{
Effects of Zeeman splitting on spin density waves
}

\author{
A. Bjelic, D. Zanchi
}

\section{To cite this version:}

A. Bjelic, D. Zanchi. Effects of Zeeman splitting on spin density waves. Journal de Physique IV Proceedings, 1993, 03 (C2), pp.C2-323-C2-326. 10.1051/jp4:1993266 . jpa-00251349

\section{HAL Id: jpa-00251349 https://hal.science/jpa-00251349}

Submitted on 1 Jan 1993

HAL is a multi-disciplinary open access archive for the deposit and dissemination of scientific research documents, whether they are published or not. The documents may come from teaching and research institutions in France or abroad, or from public or private research centers.
L'archive ouverte pluridisciplinaire HAL, est destinée au dépôt et à la diffusion de documents scientifiques de niveau recherche, publiés ou non, émanant des établissements d'enseignement et de recherche français ou étrangers, des laboratoires publics ou privés. 


\title{
Effects of Zeeman splitting on spin density waves
}

\author{
A. BJELIŠ and D. ZANCHI
}

Department of Physics, Faculty of Science, University of Zagreb, P.O. Box 162, 41001 Zagreb, Croatia

\begin{abstract}
Starting from the Hubbard model $\dot{w}$ ith the repulsive Coulomb interaction and imperfectly nested Fermi surfaces we calculate the total, i.e. spin and charge density wave, $2 k_{F}$-susceptibility above the critical temperature, taking into account both, orbital and spin coupling of electrons to the external magnetic field $\mathbf{H}$. The influence of the magnetic field on the collective modes, and in particular the emerging splitting of degeneracies present in the isotropic case $(\mathbf{H}=0)$, are also discussed.
\end{abstract}

\section{Introduction}

The external magnetic field $\mathbf{H}$ influences the spin density wave (SDW) order through the orbital and Pauli coupling. The orbital coupling may lead to the field induced stabilization of SDW [1 - 3], or to the increase of critical temperature in systems with SDW already existing at $H=0$ [4]. Furthermore, this mechanism also causes the decrease of the transverse correlation lengths (even if the nesting is perfect)[5] and the angular resonances of Lebed's [6] type whenever the magnetic lengths in the two transverse directions are commensurate $[7,8]$. These effects were mostly observed in SDW Bechgaard salts, although the orbital mechanism does not distinguish SDW from charge density wave (CDW) systems. More precisely, it does not lift the orientational degeneracy of spin in the perfect SDW order without internal spin anisotropies. This is however not the case with the Pauli coupling which introduces the Zeeman splitting in the electronic spin orientations. At first, it favors the spin orientation of SDW in the plane perpendicular to $\mathbf{H}$ [9], so that the SDW order takes place in this plane whenever the external field is stronger than the internal spin flop field. This has immediate consequences on six SDW collective modes. Within the mean-field approximation and in the isotropic $(\mathbf{H}=0)$ case they are degenerate above the critical temperature $T_{c}$. At $T<T_{c}$ three of them acquire gaps, while another three are acoustic Goldstone modes representing two spin wave and one phason branches. The finite $H$ at $T>T_{c}$ shifts two of six modes upwards. Below $T_{c}$ the spin wave mode representing the fluctuations of the spin component perpendicular to the easy plane acquires a gap proportional to the magnetic field. In this work we analyse these effects quantitatively by calculating the RPA susceptibility at $T>T_{c}$ and the corresponding lowest order harmonic term in the Landau free energy. 


\section{RPA Susceptibility}

In the discussion of all collective modes it is necessary to take into account that the SDW is a complex three-dimensional vector. Its three components are

$$
M_{i}=\Psi^{\dagger}\left[\begin{array}{cc}
0 & \sigma_{i} \\
0 & 0
\end{array}\right] \Psi, \quad i=1,2,3
$$

where $\sigma_{i}$ are Pauli matrices and $\Psi$ is the four-component fermion field

$$
\Psi^{\dagger}=\left(\Psi_{+\uparrow}^{\dagger}, \Psi_{+\downarrow}^{\dagger}, \Psi_{-\uparrow}^{\dagger}, \Psi_{-\downarrow}^{\dagger}\right)
$$

with the first index denoting two sheets of the quasi-one-dimensional Fermi surface. Furthermore, the Zeeman spitting causes the mixing of the SDW component parallel to $\mathbf{H}$ (chosen to be $M_{3}$ ) and the CDW amplitude

$$
C \equiv M_{4}=\Psi^{\dagger}\left[\begin{array}{ll}
0 & I \\
0 & 0
\end{array}\right] \Psi .
$$

The full $2 k_{F}$ - susceptibility above $T_{c}$ is thus represented by the $4 \times 4$ matrix with the components

$$
\chi_{i j}=\left\langle T_{\tau} M_{i} M_{j}\right\rangle \quad, \quad i, j=1, \ldots, 4 .
$$

The straightforward calculation of $\chi_{i j}$ within RPA for the usual Hubbard model with the imperfect nesting leads to the expression

$$
\left[\chi_{i j}\right]=\left(\begin{array}{cccc}
\frac{\chi^{\circ}(q)}{1-U \chi^{\circ}(q)} & 0 & 0 & 0 \\
0 & \frac{\chi^{\circ}(q)}{1-U \chi^{\circ}(q)} & 0 & 0 \\
0 & 0 & \frac{\chi_{g}\left(\sqrt{1+\delta^{2}}+U x_{g}\right)}{1-U^{2} \chi_{g}^{2}} & \frac{\chi_{g} \delta}{1-U^{2} \chi_{g}^{2}} \\
0 & 0 & \frac{\chi_{g} \delta}{1-U^{2} \chi_{g}^{2}} & \frac{\chi_{g}\left(\sqrt{1+\delta^{2}}-U \chi_{g}\right)}{1-U^{2} x_{g}^{2}}
\end{array}\right)
$$

Here $\chi_{g}=\sqrt{\chi_{\uparrow}(\mathbf{q}) \chi_{\downarrow}(\mathbf{q})} ; \delta=\left[\chi_{\uparrow}(\mathbf{q})-\chi_{\downarrow}(\mathbf{q})\right] / 2 \chi_{g}$ and $\chi_{\uparrow, \downarrow}=\chi^{\circ}\left(q_{1} \pm 2 \eta\right)$, where $q_{1}$ denotes the wave number in the chain direction and $\eta \equiv \mu_{B} H / v_{F}$ ( $\mu_{B}$ - Bohr magneton, $v_{F}$ - Fermi velocity). $\chi^{\circ}$ is the scalar Hartree-Fock (bubble) susceptibility which contains only orbital contributions from the magnetic field [7];

$$
\begin{aligned}
\chi^{\circ}(\mathbf{q}, \omega)= & \int d^{3} q^{\prime} \int d y \chi_{1 \mathrm{dim}}^{o}\left(q_{1}^{\prime}, \omega\right) \\
& \exp \left\{i\left[\left(q_{1}^{\prime}-q_{1}\right) y+\Omega_{+}\left(y, \mathbf{q}_{\perp}^{\prime}\right)-\Omega_{+}\left(0, \mathbf{q}_{\perp}^{\prime}\right)-\Omega_{-}\left(y, \mathbf{q}_{\perp}^{\prime}+\mathbf{q}_{\perp}\right)+\Omega_{-}\left(0, \mathbf{q}_{\perp}^{\prime}+\mathbf{q}_{\perp}\right)\right]\right\}
\end{aligned}
$$

with $\chi^{\circ}(\mathbf{q}, \omega)$ being the one-dimensional bubble. The "orbital" phases are given by

$$
\Omega_{\alpha}\left(y, \mathbf{q}_{\perp}\right) \equiv \sum_{\vec{\delta}_{\perp}} \frac{\alpha t_{\delta}}{v_{F} i\left(\mathbf{A}^{\prime} \vec{\delta}_{\perp}\right)} \exp \left[i\left(\alpha \mathbf{Q}+\mathbf{q}_{\perp}-\mathbf{A}\right) \vec{\delta}_{\perp}\right] \quad, \quad \alpha=+,-
$$

where $2 Q=\left(2 k_{F}, Q_{b}, Q_{c}\right)$ denotes the SDW wave vector and the vector potential is $\mathbf{A}=(0, H \cos \theta,-H \sin \theta) y \equiv \mathbf{A}^{\prime} y$, with $\theta$ denoting the angle between $\mathbf{H}$ and the c-axis in the $(b, c)$ plane. The summation in eq.(7) goes over the transverse neighbors $\vec{\delta}_{\perp}$ with the hopping integrals $t_{\delta}$. As is evident from eq. (5) that the Zeeman splitting does not affect the SDW susceptibility in the plane perpendicular to $\mathbf{H}$, given by $\chi_{11}=\chi_{22}=\chi^{\perp}$. The eigenvalues of $\left[\chi_{i j}\right]$ in the subspace $\left(M_{3}, C\right)$ are

$$
\chi_{ \pm}^{\|}=\frac{\chi_{g}}{\sqrt{1+\delta^{2}} \pm \sqrt{U^{2} \chi_{g}^{2}+\delta^{2}}} .
$$


$\chi$ - is the branch which for $U>0$ reduces to $\chi_{33}$ in the limit $|\mathbf{H}| \rightarrow 0$ Its poles are therefore those SDW collective modes which are renormalized due to the Zeeman splitting. Note also that in the long-wavelength limit $M_{3}$ and $C$ are decoupled, since $\delta(q=0)=0\left(\right.$ but $\chi_{s}(\mathbf{q}=0) \neq \chi_{0}(\mathbf{q}=0)$ ). Comparing $1 / \chi^{\perp}$ and $1 / \chi_{-}^{\|}$, it comes out that, irrespectively of the value of $H$, the absolute minimum of $1 / \chi^{\perp}$ at $\mathbf{q}=0$ is at all temperatures higher than the absolute minimum of $1 / \chi_{-}^{\|}$ (which can be at a finite value of $q$ for $\mu_{B} H \geq 0.34 \cdot 2 \pi T_{c}$ ). The mean-field critical temperature is therefore not affected by the Zeeman splitting.

\section{Collective Modes}

Let us consider the simplest case of the perfect one-dimensional nesting (all $t_{\delta}$ 's equal to zero). The long-wavelength propagator of the two degenerate modes from $\chi_{-}^{\|}$is then given by the expansion

$$
\chi^{\|}-\left(q_{1}, \omega\right) \simeq\left(i \alpha \omega+a^{\|}+\xi_{1}^{\|} q_{1}^{2}\right)^{-1}
$$

where $\sqrt{a^{l l}}$ is proportional to the gap at $q_{1}=0, \alpha \approx \pi / 8 T$ and $\xi_{1}^{\|}$is the longitudinal correlation length. The dependence of these coefficients on $h \equiv \mu_{B} H / 2 \pi T$ at $T=T_{c}$ and for $N_{F} U=0.16$ is shown in Fig.1. $a^{\perp}$ and $\xi_{1}^{\perp}$ are the corresponding quantities from the $\chi^{\perp}$ - dispersion $\left(a^{\perp}=0\right.$ at $T=T_{c}$ ). The value $a^{\| l}$ depends parabolically on the magnetic field up to $h=h_{c}=0.34$. At this value of $h$ the longitudinal correlation length $\xi_{1}^{l}$ approaches zero, signifying that by a further increase of the magnetic field the maximum of $\chi_{-}^{\|}$shifts to the finite point $q=\kappa$ with $\kappa \sim \sqrt{h-h_{c}}$, with the asymptotic behavior $v_{F} \kappa / 4 \pi T=h$. In Fig.1 we also show $a^{l l}$ and the correlation length measured with respect to the new maximum at $h>h_{c}$.

Some hints on the collective modes below $T_{c}$ may be drawn from the Landau expansion for the free energy. Expecting that main effects of Zeeman splitting enter through the second-order term (i. e. the susceptibility (5)), we complete this expansion by the fourth-order term from Ref.10. This term introduces an important constraint on the SDW order in the isotropic $(\mathbf{H}=0)$ case, namely all three components $M_{i}$ have a common phase in the equilibrium. As a consequence, the six collective modes split into three Goldstone branches mentioned in the Introduction and three modes with the gaps. One of the latter modes is the usual amplitudon, while the remaining two are the fluctuations of the relative phases with respect to their zero values imposed by the above constraint. The Zeeman splitting introduces a finite gap into the Goldstone mode representing fluctuations of the spin orientation out of the easy plane. The gap of this mode is determined by $\sqrt{a^{\perp}-a l l}$. Another mode influenced by the Zeeman splitting is associated to the fluctuations of the phase of $M_{3}$ with respect to the common phase of the order parameter. Here the dependence on $\mathbf{H}$ enters mainly through the correlation length $\xi_{1}^{\|}$.

The finite transverse hoppings do not alter significantly the results of Fig.1, and vice versa, the effects of Zeeman splitting on the transverse correlation lengths $\xi_{2,3}^{\|}$of $\chi_{-}^{\|}$- modes are weaker than those originating from the orbital contribution (6) [5,11]. Finally, the imperfect nesting brings a new type of angular resonances [12] in the gap and the correlation lengths of all modes influenced by the Zeeman splitting. They appear at the angles $\cos \theta=4 \mu_{B} H / n \omega_{b}$ ( $\mathrm{n}$ - integer), as the combined effect of Pauli and orbital coupling. The quantitative analysis of these resonances will be given elsewhere [11]. 
(a)

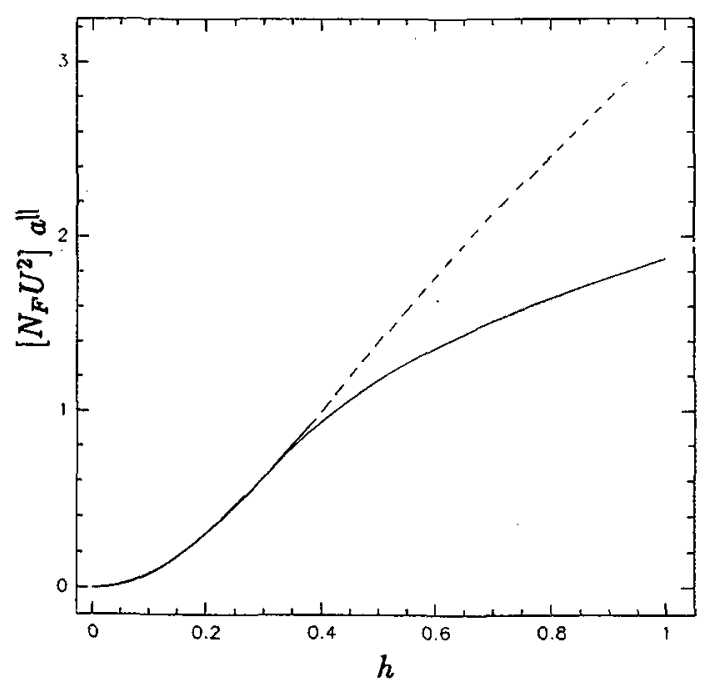

(b)

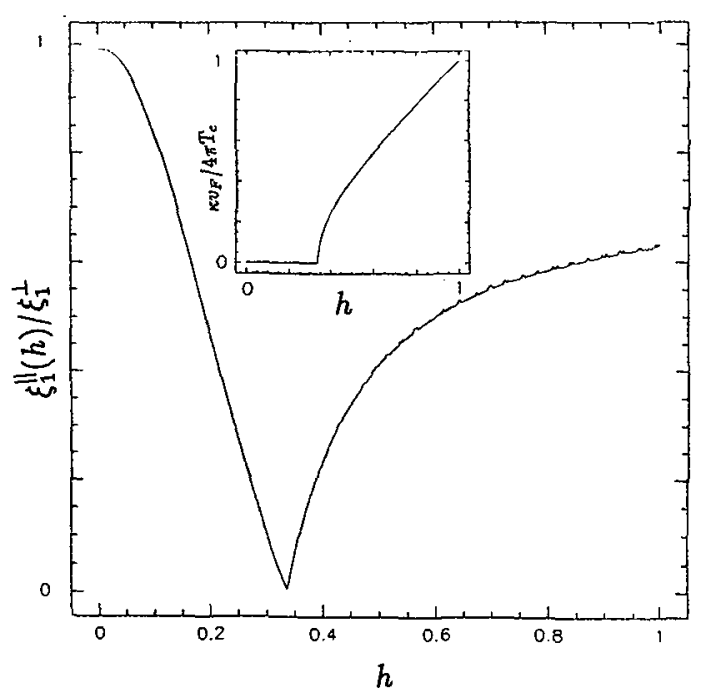

Fig.1. (a) all at $T=T_{c}$ as the function of $h$. The lower curve at $h>h_{c}$ represents $a^{\| l}(\kappa)$.

(b) The corresponding ratio $\xi_{1}^{\|}(h) / \xi_{1}^{\perp}$. The insert represents the dependence of $\left(v_{F} / 4 \pi T_{c}\right) \kappa$ on $h$.

\section{References}

[1] GOR'KOV L. P. and LEBED' A. G., J. Physique Lett.45 (1984) L433..

[2] HERITIER M., MONTAMBAUX G. and LEDERER P., J. Physique Lett.45 (1984) L943.

[3] MAKI K., Phys. Rev.B 33(1986) 4826.

[4] BJELIŠ A. and MAKI K., Phys. Rev.B 42 (1990) 10275.

[5] BJELIŠ A. and MAKI K., Phys. Rev.B 44 (1991) 6799.

[6] LEBED' A. G., Pisma Zh.Eksp.Teor.Fiz.43 (1986) 137 [JETP Lett. 43 (1986) 174].

[7] BJELIŠ A. and MAKI K., Phys. Rev.B 45 (1992) 12887; Synth. Metals 55-57 (1993) 2749.

[8] SUN Y. and MAKI K., preprint.

[9] POILBLANC D. and LEDERER P.,Phys. Rev.B 37 (1988) 9650.

[10] HASEGAWA Y. and FUKUYAMA H., J. Phys. Soc. Japan 55 (1986) 3978.

[11] BJELIŠ A. and ZANCHI D., to be published.

[12] LEBED' A. G., J. Physique 2 (1992) no 11. 